\title{
Considering Diversity in Spatial Decision Support Systems
}

\author{
Ningchuan Xiao \\ Department of Geography \\ The Ohio State University, Columbus, OH 43210 \\ Telephone: +1-614-292-4072 \\ Fax: +1-614-292-6213 \\ Email: xiao.37@osu.edu
}

\section{Introduction}

Spatial decision problems are often computationally intensive to solve. For many problems that have multiple objectives, there may not exist a single solution that is deemed to be optimal by all stakeholders. To address such decision problems, a variety of solution approaches have been developed. The literature, however, seems to be less concerned with how to incorporate these approaches such that a better solution can be ultimately reached. A more interesting question is, if existing solution approaches can be collectively used to provide high-quality solutions, is it worth to develop new ones? Moreover, how can we successfully incorporate different perspectives of decision makers and stakeholders to generate more robust and reliable solutions that are satisfactory to a wider group of people?

The above questions are related to an interesting topic in social science: diversity, referring to a state of difference exhibited in a system and its components. Recent developments have demonstrated that diversity may provide better solutions to highly complex problems in social and economic domains such as long-term prediction (Page, 2007). The purpose of this paper is to discuss how the concept of diversity manifests in spatial decision making. In the remainder of this paper, I first identify the kinds of diversity in spatial decision making, and then discuss a number of approaches to incorporating diversity into geographical problem solving.

\section{Kinds of Diversity}

Three steps are typically required to complete a (spatial) decision making process (Simon, 1960). Starting at the intelligence step, the problem must be formulated so that alternative solutions can be found in the second step called design. In the third step called choice, a final decision must be made based on the alternatives identified. Diversity is ubiquitous in all steps. For example, diversity occurs when the problem is interpreted and formulated by different stakeholders from different perspectives, solved using different methods, and presented to decision makers who have different preferences. Specifically in this paper, I group diversity into four categories, according to the context in which diversity occurs.

- Diversity of the solution space. The solution space of a decision problem is formed by all possible solutions to the problem. For many spatial decision problems, it is often intuitive to consider a solution space as a map space, or geographical space, which contains all the maps, each representing a solution to the problem. The diversity of the solution space for a 
spatial decision problem refers to the multiplicity of the solutions, especially in terms of the spatial configuration.

- Diversity of the objective space. As a dual space of the solution space of a problem, the objective space is a multidimensional space formed by a set of coordinates, each corresponding to an objective of the problem. These objectives reflect different, often conflicting goals. A consequence of such difference is the trade-off among alternative solutions, meaning there is no single solution that can be considered to be satisfactory with respect to all goals.

- Diversity of optimality. A fundamental problem of (spatial) decision making is that the decision problem may be ill-structured because many social, economic, and environmental factors are difficult to be included in problem formulation. This feature suggests that the optimality obtained based on the original problem formulation may become sub-optimal when new factors are considered.

- Diverse toolboxes. Two main types of solution approaches to solving spatial decision problems exist. The first type of approach is called exact methods that can guarantee to find global optimal solutions to the problems, though they may not be efficient for problems with large input sizes. The second type of approach, called heuristics, are often more efficient, though they do not guarantee the global solutions to be found. For each decision problem, there is often a set of different heuristics developed.

\section{Incorporating Diversity}

Diversity can be incorporated into spatial decision making processes in a variety of ways. Here, I identify a number of technologies that can be used to promote or utilise diversity discussed above for the purpose of spatial decision making.

Solution approaches developed in the literature can be used in different fashion. Though the common way of using these methods independently is useful, the overall performance can be improved if these methods are used collectively. Table 1 illustrates this point based on solving the $p$-median problem using two different approaches: a method called TB developed by Teitz and Bart (1968), and a method called SA that is based on simulated annealing (Kirkpatrick et al., 1983). In addition to running these two methods independently, four strategies were also used. The relay strategy uses TB first and then the solution found by TB is used in SA. The consensus strategy is similar to relay, but the solution found by SA will be used in TB and so on, until no improvement can be made. To use the compete strategy, both TB and SA start independently and then, during each iteration of both methods, the current solutions are compared and the winner will be used by both method for the next iteration; the process continues until no improvement can be made. The collaborate strategy depends on an exchange mechanism such that the two methods exchange their current solutions during each iteration.

Forty benchmark $p$-median problems (Beasley, 1985) were used to test these strategies. Each strategy was run 20 times, with the best, worst, and average results of these runs reported in Table 1. The results clearly suggest that some strategies (e.g., collaborate) consistently outperform the independent use of TB or SA, while some strategies (e.g., compete) may not necessarily outperform the original two methods. 
Table 1: Solving the $p$-median problem using two methods

\begin{tabular}{llrrrrrr}
\hline & Method & TB & SA & Relay & Consensus & Compete & Collaborate \\
\hline Best case & Hits & 23 & 31 & 30 & 33 & 31 & 34 \\
& Better than TB/SA & & & 3 & 4 & 5 & 5 \\
& Tied with TB/SA & & & 31 & 35 & 30 & 33 \\
& Worse than TB/SA & & & 6 & 1 & 5 & 2 \\
\hline Average & Hits & 16 & 9 & 16 & 14 & 9 & 19 \\
& Better than TB/SA & & & 14 & 20 & 10 & 23 \\
& Tied with TB/SA & & & 14 & 15 & 8 & 17 \\
& Worse than TB/SA & & 12 & 5 & 22 & 0 \\
\hline Worst case & Hits & 16 & 9 & 16 & 14 & 9 & 19 \\
& Better than TB/SA & & & 13 & 17 & 8 & 20 \\
& Tied with TB/SA & & & 19 & 18 & 12 & 20 \\
& Worse than TB/SA & & 8 & 5 & 20 & 0 \\
\hline
\end{tabular}

Extending the above experiment, we can consider each method as an agent that is equipped with a particular skill of solving some problems. An agent-based modelling framework, therefore, can be regarded as a platform to utilise the diversity of toolboxes in spatial decision making. In addition to such toolbox perspective, agent-based models can also incorporate multiple players (decision makers) that have different belief systems and reflect different preferences to the decision problem. Simulation results of these models can be used by decision makers to learn interesting system behaviours.

Finally, it is important to note that evolutionary computation can be used to generate a diverse set of solutions to a decision problem and visualisation techniques can be used to help decision makers examine these solutions (Xiao et al., 2007). In a broader context, public participation geographical information systems can provide a platform to encourage a wide range of stakeholders to participate a decision making process (Sieber, 2006).

\section{Discussion and Conclusions}

Considering diversity in spatial decision support systems is consistent with the postmodernist view (see, for example, Macmillan, 1997) that adds to a computationally sophisticated environment of geocomputation. From a social or political point of view, promoting diversity in the decision process is a step closer toward a more appealing democratic process. It will be an interesting debate to see if such an effort will provide us "better" decisions, the meaning of which may be beyond its methodological domain and of course is another aspect of diversity.

\section{References}

Beasley, J. E. (1985). A note on solving large p-median problems. European Journal of Operational Research 21, 270-273.

Kirkpatrick, S., C. D. Gelatt, and M. P. Vecchi, Jr. (1983). Optimization by simulated annealing. Science 220, 671-680.

Macmillan, B. (1997). Computing and the science of geography: the postmodern turn and the geo- 
computational twist. In Proceedings of the 2rd International Conference on GeoComputation. Otago, New Zealand: University of Otago. CD-ROM.

Page, S. E. (2007). The Difference: How the Power of Diversity Creates Better Groups, Firms, Schools, and Societies. Princeton, NJ: Princeton University Press.

Sieber, R. (2006). Public participation geographic information systems: a literature review and framework. Annals of the Association of American Geographers 96(3), 491-507.

Simon, H. A. (1960). The New Science of Management Decision. New York: Happer \& Row.

Teitz, M. B. and P. Bart (1968). Heuristic methods for estimating the generalized vertex median of a weighted graph. Operations Research 16, 955-961.

Xiao, N., D. A. Bennett, and M. P. Armstrong (2007). Interactive evolutionary approaches to multiobjective spatial decision making: A synthetic review. Computers, Environment and Urban Systems, forthcoming. 